\title{
Research Priorities of Clinical Nurses and Midwives in the Eastern Mediterranean Region: a Mixed Methods Study
}

\author{
Carolyn J. Sun ${ }^{1,2, *}$, Caroline J. Fu ${ }^{1}$, Roa Altaweli ${ }^{3, \dagger}$, Salem Al Touby ${ }^{4}$, Cheherezade Ghazi ${ }^{5}$, Maaly Guimei ${ }^{6}$ \\ ${ }^{1}$ Department of Scholarship and Research, Columbia University, School of Nursing, New York, USA \\ ${ }^{2}$ Department of Nursing Innovation and Research, New York-Presbyterian Hospital, New York, USA \\ ${ }^{3}$ Obstetrics and Gynecology Department, East Jeddah Hospital, Jeddah, Saudi Arabia \\ ${ }^{4}$ Nursing Program at Oman College of Health Science, Ministry of Health Oman, Muscat, Oman \\ ${ }^{5}$ Department of Community Health Nursing, Badr University, Cairo, Egypt \\ ${ }^{6}$ Community Health Nursing Department, Faculty of Nursing, Alexandria University, Alexandria, Egypt
}

ARTICLE IN F O
Article History
Received 23 February 2019
Accepted 3 March 2019
Keywords
Eastern Mediterranean Region
clinical staff
Middle East
nursing
research
priorities

\begin{abstract}
Effective use of resources in healthcare research is essential in meeting the United Nation's Sustainable Development Goal (SDG) 3 to achieve universal health coverage, increase retention of healthcare workers, and strengthen the capacity of all countries to reduce risk and manage global health risks; the World Health Organization (WHO) also identifies nursing research as an important piece of the framework for improving global healthcare. Determining research priorities to reduce redundancy and ensure a solid evidence base for practice is especially critical in resource-limited countries or those facing healthcare crises such as those in the Middle East. To identify regional research priorities for nursing, focus group discussions composed of hospitalbased nurses were conducted in Egypt, Oman, and Saudi Arabia. Forty-eight percent of research priorities were nearly the same as those identified as critical by regional nursing leadership in a previous study, demonstrating consistency between clinicianand administrator-identified research priorities, and suggesting healthcare administrators are well attuned to the research needs of clinicians. Both groups identified critical gaps in population and community health research. Across countries, research priorities identified were related to nursing workforce, health systems research, and quality of care, representing critical issues needing investigation to build a solid evidence base for nursing practice.
\end{abstract}

(c) 2019 Atlantis Press International B.V.

This is an open access article distributed under the CC BY-NC 4.0 license (http://creativecommons.org/licenses/by-nc/4.0/).

\section{INTRODUCTION}

With a global shortage of healthcare providers, particularly nurses and midwives, effective use of healthcare resources and research is essential in meeting the United Nation's Sustainable Development Goal (SDG) 3 to achieve universal health coverage, increase retention of healthcare workers, and strengthen the capacity of countries to reduce and manage global health risks $[1,2]$. As such, nurses and midwives are essential to strengthening the core of healthcare [3]. To do this, nurses and midwives must conduct relevant and timely research; setting priorities is an important first step to ensure appropriate topics are researched and to reduce redundancy [2].

Nurses require advanced training and supporting evidence to meet increasingly challenging healthcare demands [4]. Compounding the worsening nursing shortage within the Eastern Mediterranean Region (EMR) [3] are a myriad of complicating factors like social turmoil, political disorder, and unparalleled mass emigration and immigration that necessitate both an exploration into the healthcare needs of refugee populations and a re-examination of nursing research priorities [5]. Building a solid evidence base for nurses and

"Corresponding author. Email: cjs2200@columbia.edu

${ }^{\dagger}$ Present Address: Ministry of Health Saudi Arabia, Nursing Affairs General Department, Midwifery Department, Saudi Arabia midwives requires a strong regional nursing research program with expertise to publish and disseminate new knowledge. Considering the nursing shortage and scarcity of clinically focused nursing and midwifery research, achieving these objectives requires a unified effort [6,7]. Furthermore, nurses and midwives must themselves establish regional priorities to produce research relevant to address the population's needs, as outside funders often set priorities [8].

The EMR, one of six World Health Organization (WHO) member state regions, includes Afghanistan, Bahrain, Djibouti, Egypt, the Islamic Republic of Iran, Iraq, Jordan, Kuwait, Lebanon, Libya, Morocco, the Occupied Palestinian territory, Oman, Pakistan, Qatar, Saudi Arabia, Somalia, Sudan, Syrian Arab Republic, Tunisia, United Arab Emirates, and Yemen [9] (Figure 1). Regionally, midwives and nurses have varying levels of required education and training but are often categorized within the same profession. Both are frontline healthcare providers well poised to determine clinical research needs [10-12].

Nursing research has increasingly become a marker for nursing and hospital quality with an emphasis on nurse engagement; Magnet recognition for nursing excellence in hospitals requires ongoing internal research and changes to practice based on nurse-generated evidence $[11,13]$. Setting clear, focused priorities is considered a vital first step in research [2], and it is increasingly understood that clinical nursing staff are essential to this process, yet, to date we 


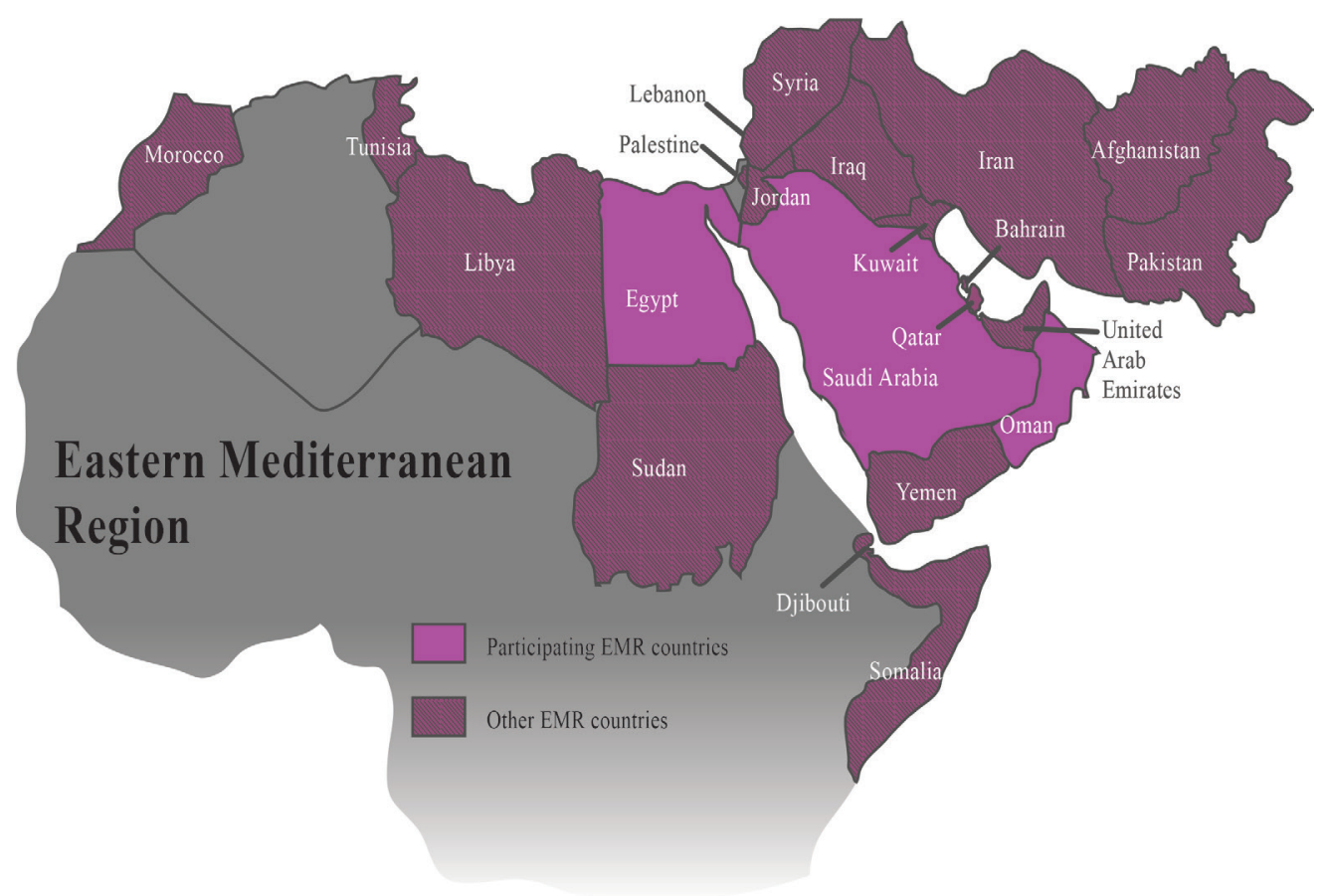

Figure 1 Countries in the Eastern Mediterranean Region that participated in focus group discussions to determine regional critical nursing research priorities.

have found no evidence of clinical nurses' and midwives' involvement in developing a regional research agenda in the EMR [14].

To address this, we previously conducted a scoping review of regional nursing and midwifery clinical research [12] and a Delphi survey to assess research priorities from the perspectives of nursing leadership [14]. Regional leaders then gathered at a research summit in July 2016 to discuss results of these studies. They agreed it would be of paramount importance to engage nurses currently providing bedside care to give additional insight into research needs and help connect clinical and academic silos.

The objectives of this study were to use Focus Group Discussions (FGDs) to (1) understand what nurses and midwives currently practicing in the acute care setting in the EMR feel are critical research priorities; and (2) compare the results with priorities determined by regional nursing leadership and existing EMR nursing literature.

\section{MATERIALS AND METHODS}

\subsection{Study Design}

A mixed methods approach was used. First, FGDs were used to explore nursing and midwifery clinical research priorities for the region $[15,16]$. Secondly, a quantitative approach was used to compare the FGD results with two previous studies: a Delphi survey and a scoping review (described in Section 1) [12,14]. Qualitative FGD results were reported following COnsolidated criteria for REporting Qualitative research (COREQ) [17].

\subsection{Ethical Considerations}

From the summit (described above), nurse scientists (NSs) in Egypt, Oman, and Saudi Arabia agreed to host FGDs (Figure 1) and obtained Institutional Review Board (IRB) approval at their respective sites as did the principal investigator (PI) at the coordinating institution, Columbia University School of Nursing (IRB approval number AAAR1924).

\subsection{Qualitative Methods: FGDs}

\subsubsection{Development of questioning route}

Using web-based technology to facilitate international collaboration, a regionally appropriate, culturally sensitive FGD script was developed for use across countries to elicit clinical nurses' and midwives' perspectives on research priorities using the methods outlined by Morgan and colleagues [15]. Following development, one team member translated the script into colloquial Arabic; it was iteratively edited until consensus was reached.

\subsubsection{Sample and setting}

Each NS secured a meeting room (either at the NS's university or the hospital where nurses were recruited) and posted flyers and/ or recruited via word of mouth using both convenience and snowball sampling, aiming to recruit eight (minimum six, maximum 12) participants. NSs briefly screened interested individuals against the eligibility criteria (practicing nurse or midwife with at least 2 years of experience in their current setting). Each NS obtained a recording device and recruited one research assistant (RA) to help take notes, operate the recording device, and ensure that each participant completed the consent form and screening questionnaire. Each FGD was scheduled for $60 \mathrm{~min}$ and was facilitated in Arabic, English, or both by the NS, who was both a registered nurse (RN) and/or midwife and a doctorally prepared local nursing leader; these NSs were female except in Oman where the NS and RA were male. There were no dropouts once recruited. 


\subsubsection{Data analysis}

After each FGD, the NS and RAs held a debriefing session. The recordings were uploaded to a password protected web-based sharing site, DatAnywhere File Sharing, then downloaded, translated, and transcribed. The coordinating PI and another RA analyzed all FGDs using framework analysis [15,16] of line-numbered paper transcripts; coding discrepancies were discussed until consensus was reached. Codes and themes derived thereof were indexed and a codebook was developed. Each statement, sentence or paragraph was coded with as many codes as necessary to accurately describe all topics presented and results were confirmed with NSs who led the FGDs.

\subsubsection{Rigor and trustworthiness}

To build rapport with participants, the facilitators introduced themselves and the research goal and asked one introductory question (Appendix A, Question 1). No nonparticipants were present, and no repeat interviews were conducted. Due to the nature of the study (broad geographical location and translation and analysis of transcripts), it was not possible to return the transcripts to the participants. Participants' answers were restated back to them for member checks, and the two coders coded the data and discussed themes until consensus was reached [18].

\subsection{Quantitative Methods}

Inter-rater reliability between coders was assessed by Cohen's kappa [19] using IBM SPSS version 23.0.2 (IBM Corp, released 2015. IBM SPSS Statistics for Mac, Version 23.0.2. Armonk, NY: IBM Corp.). Indexed codes were then mapped and interpreted for quantitative data abstraction [16]. Additional quantitative information (frequency of codes per country) was compared between countries [15] and against the Delphi survey results [14] and topics in the regional literature from the scoping review [12].

\section{RESULTS}

A total of 38 codes (Appendix B) and four themes (Table 1) were developed. Cohen's kappa for inter-rater agreement of $0.775(p<0.001)$ was achieved, indicating substantial agreement [19]. Appendix B presents the frequency of codes. Over 30 pages of transcripts from four FGD sites were reviewed: two in Egypt (12 participants per group), one in Saudi Arabia (six participants) and one in Oman (12 participants). The Egypt and Oman FGDs were conducted through a coordinating site with participants currently practicing in various acute care units at public and private regional hospitals; the Saudi Arabia FGD was conducted at a single public hospital.

\subsection{Themes and Codes}

Four themes emerged in analyzing the FGD transcripts: nursing shortages, Health Systems Research (HSR), patient care, and knowledge gaps (Table 1).
Table 1 Themes overarching all focus group discussions

\begin{tabular}{|c|c|c|}
\hline Themes & $\begin{array}{l}\text { Examples of } \\
\text { codes informing } \\
\text { this theme }\end{array}$ & Description \\
\hline $\begin{array}{l}\text { Nursing } \\
\text { shortage }\end{array}$ & $\begin{array}{l}\text { Nursing } \\
\text { workforce } \\
\text { Health } \\
\text { systems }\end{array}$ & $\begin{array}{l}\text { Shortages in human resource support, } \\
\text { supplies, facilities, and across other } \\
\text { sectors indicated by discussions on } \\
\text { burnout, workload, safety, and lack of } \\
\text { education and training. }\end{array}$ \\
\hline $\begin{array}{l}\text { Knowledge } \\
\text { gap }\end{array}$ & $\begin{array}{l}\text { Knowledge } \\
\text { deficit } \\
\text { Skill deficit }\end{array}$ & $\begin{array}{l}\text { Although not always specifically } \\
\text { mentioned, discussions often indicated } \\
\text { unfamiliarity with current literature or } \\
\text { how to obtain specific evidence on a } \\
\text { topic. Lack of skill for specific nursing } \\
\text { tasks also arose frequently. }\end{array}$ \\
\hline $\begin{array}{c}\text { Patient } \\
\text { care }\end{array}$ & $\begin{array}{l}\text { Nutrition } \\
\text { Wound care } \\
\text { Geriatrics } \\
\text { Obesity } \\
\text { Medication }\end{array}$ & $\begin{array}{l}\text { Over } 70 \% \text { of the codes }(27 / 38) \text { referred } \\
\text { to patient care and patient-related } \\
\text { outcomes. }\end{array}$ \\
\hline $\begin{array}{l}\text { Health } \\
\text { systems } \\
\text { research }\end{array}$ & $\begin{array}{l}\text { Health } \\
\text { systems } \\
\text { Nursing } \\
\text { workforce }\end{array}$ & $\begin{array}{l}\text { Many topics pointed to larger systems, } \\
\text { e.g., restructuring nursing education } \\
\text { levels, formalizing a system to rotate } \\
\text { nurses between primary and tertiary } \\
\text { practice settings. }\end{array}$ \\
\hline
\end{tabular}

\subsubsection{Nursing shortages}

Within the theme of nursing shortages, participants across all groups most frequently discussed nursing workforce issues, including staffing shortages, task management, duties, shifts, and burnout; workforce issues were also identified as a critical priority. A nurse in Egypt stated "The nursing team is severely suffering, receives low salaries, no recognition, and is stressed and stretched out in work. We need research on strategies to relieve workload on nurses, focus on how they can receive better education on how to work in certain work environments, how to increase interest in work and receive salaries [that] ensure a good living standard." A nurse in Oman stated, "Shortage and absenteeism in the unit affects staff who are giving care for the baby because their workload increases." Another in Saudi Arabia said, "There are many factors which may cause the nurse to become stressed, and this stress affects the patients."

Nurses' mental health was discussed in all groups, and participants in Egypt and Saudi Arabia also discussed the public perception of nursing. One Egyptian nurse said, "Nurses are considered the lowest level, lowest profession. Why don't they enhance the image of nursing in the media? ... Media always attacks nursing... saying that doctors are on top and nurses are the lowest of the low."

\subsubsection{Health systems research}

Health systems research, a budding, interdisciplinary field that examines how health systems and policies affect other determinants of health [20], also permeated the discussions. Over a quarter of the codes (e.g., health systems research, national policy, lack of resources, and infrastructure) fell directly under 
this theme. Participants across all FGDs described situations in which roles were unclear when handling or transferring patients. An Intensive Care Unit (ICU) nurse in Egypt said, "When there is a new admission all of our staff working in the ICU work without any guidelines to follow. There is nothing to identify the role of everyone on the team. Without a team, the work is disorderly." Similarly, a nurse in Oman said, "There is no coordination...[for transitioning a] baby to go from [neonatal ICU] NICU to [pediatric ICU] PICU." Health team workflow was identified as a critical research priority in all FGDs and was discussed most extensively in Egypt.

Other systems-level issues included protocols, infrastructure, and technology, systemic strain from lack of resources like access to clean water and a secure supply chain for medical supplies, and the standardization of nursing practice and training. Nurses in Egypt discussed restructuring nursing education levels or formalizing a system to rotate nurses between primary and tertiary practice settings.

\subsubsection{Patient care}

Over $70 \%$ of the coded priorities described specific areas of patient care, which varied by FGD as nurses often referenced their direct experiences.

Quality of care including safety was frequently discussed and was identified as a critical priority. A nurse in Egypt said, “...the patient can have blood and plasma transfusions on the ward, without monitors so you don't know when patient is having a reaction. If we knew, something could be done but so many die because of unmonitored transfusion reactions."

Other patient care areas described as critical were patient education, pain management, palliative care, and mental health. Oman FGD participants discussed care for road traffic accident patients and increasing public awareness of burn wounds from explosions at home caused by gas leaks or poorly functioning ovens. Different from other countries, Saudi Arabia FGD participants identified issues in women's health and patient education.

\subsubsection{Knowledge gaps}

Some responses from nurses and midwives suggested knowledge and skill gaps in identifying evidence-based practice and in being informed on current research on certain topics; this also was identified as an underlying theme. For example, some nurses suggested there should be testing for cervical cancer for which there are already established tests and clinical guidelines.

\subsection{Research Priorities across Themes}

Many research priorities integrated multiple themes. For example, a nurse in Oman described the provision of culturally relevant research using aspects of patient care, health systems, and infrastructure. "Recently, we started the DNR [do not resuscitate] policy according to the evidence base in other [European] countries. For our culture, Islamic culture, this is something we have to modify and research and then we have to re-apply it because the
DNR policy we have copied in our organization and applied for our patients is not the perfect way for us... we can use the evidence base but we have to study our culture in depth."

Infection control, the most frequently occurring specific research priority, simultaneously touched on a lack of resources, limitations of hospital policies, healthcare workers, education, infrastructure, and safety. "Infection control is a weak point for governmental medical entities, because of lack of resources and facilities. For example, in a governmental hospital, we used to have only three packs of gloves to use for 12 patients over a period of 12 hours... In private places the infection control policy is implemented efficiently with suitable guidelines and resources so the treatment results are good," (Egypt).

\subsection{Comparison to a Delphi Survey of Regional Nurse Leaders}

Forty-eight percent of the research topics that nurses and midwives raised closely matched responses of regional nursing experts (i.e., nursing school deans, high-ranking ministry of health officials, WHO leaders) in a recent Delphi survey [14]; 19\% were related, and $33 \%$ were different (Table 2). Although we asked for patient outcomes related topics in the FGDs, because of their nature (i.e., freely flowing conversation), other topics may have been inadvertently introduced, which could account for some differences in the responses. In both studies, we asked for critical research priorities. Some critical priorities identified by FGD participants were only considered important or moderately important in the Delphi survey (Table 2).

\subsection{Comparison to a Scoping Review}

Of the 38 topics identified, 25 (65.8\%) were not directly mentioned when compared to a recent scoping review of nursing and midwifery clinical research in the EMR (Table 2) [12]. However, among these, six (24\%) were not specific to research with patient-related outcomes, which was the focus of the scoping review. The most frequently mentioned research topics in the FGDs, nursing workforce, HSR, and quality and safety issues (also mentioned as critical in the Delphi survey), were not specifically mentioned in the scoping review. Infection prevention/control was ranked as a critical priority in the Delphi survey, and appeared in $1.0 \%$ of the literature retrieved in the scoping review. Women's health and mental health were the FGD topics that appeared most often in the scoping review ( $18.1 \%$ and $16.7 \%$ of the literature, respectively). Maternal/ child health was the most often cited topic in the scoping review but, because of the lack of frequency, topics in this category like breastfeeding (mentioned in the Saudi Arabia FGD) were not separately coded.

\section{DISCUSSION}

\subsection{FGDs: Codes and Themes}

Participating clinical nurses and midwives frequently identified research questions that immediately impacted their practice and work life. While their perspectives and priorities could be expected 
Table 2 Comparison of research topics identified as critical for the Eastern Mediterranean Region by nursing leadership in Delphi study [14] and those identified by clinical nurses in FGDs ${ }^{\mathrm{a}}$

\section{Priorities from Delphi}

Culturally competent approaches to health promotion and care

Same or similar topics in both Delphi and FGDs, $n=10(47.6 \%)$

Emergency preparedness for disasters Mental health

Infection prevention/control

Quality assurance/patient safety issues/medical errors

Road traffic accidents

Palliative care

Self-management of disease, patient participation in care

Primary health care

Obesity/bariatrics

Related topics in both the Delphi survey and FGDs, $n=4(19.0 \%)$

Different topics in Delphi and FGDs, $n=7(33.3 \%)$
Community programs linked to clinical services to ensure the management of all chronic disease (obesity prevention, etc., and epidemiology and surveillance)

Community health/community based practice/public health

Women's cancer

All noncommunicable disease risk factors (human health behavior, nutrition)

\section{Breast cancer}

Cancer

Cardiovascular disease (including coronary heart disease, stroke, and self-care management)

Chronic illness

Diabetes

Hypertension

Kidney disease

\section{Priorities from FGDs}

Culture

Emergency preparedness

Mental health

Mental health of nurses

Infection control

Quality/safety

Length of stay

Road traffic accidents

Palliative care

Patient participation in care

Patient education

Primary care

Obesity

Public awareness and safety

Women's health ${ }^{c}$

Nutrition

Access

Alarm fatigue

Bombings/explosions

Burns

Communication $^{\mathrm{b}, \mathrm{d}}$

\section{$\mathrm{CAM}^{\mathrm{b}}$}

Documentation

Geriatrics $^{\mathrm{d}}$

Health systems research ${ }^{\mathrm{d}}$

Healthcare costs ${ }^{\mathrm{b}}$

Healthcare infrastructure ${ }^{\mathrm{b}, \mathrm{d}}$

Lack of resources ${ }^{\mathrm{b}}$

Medications

Nil per os

Nursing workforce ${ }^{\mathrm{b}}$

Pain management ${ }^{\mathrm{d}}$

Pediatrics $^{\mathrm{d}}$

Policy

Public perception of nursing

Sexually transmitted

infections $^{c}$

Using evidence

Wound care
Percentage of times priority appeared in scoping review [12]

$1.9 \%^{\mathrm{b}}$

$16.7 \%^{\mathrm{b}}$

$1.0 \%$

$-$

$-$

$-$

$1.4 \%$

$9.5 \%$

$-$

$-$

$1.9 \%$

Public awareness and safety not specifically mentioned

5.2\% (Community health/disease prevention/health promotion) $18.1 \%$ (Women's health)

$-$

Neither specifically mentioned $13.8 \%$ (Cancer)

Alarm fatigue not specifically mentioned $10.0 \%$ (Cardiac disease) 2.9\% (Wars/conflict)

8.1\% (Chronic diseases)

Burns not specifically mentioned

Diabetes not specifically mentioned

(Chronic diseases (8.1\%)

Communication not specifically mentioned

Hypertension not specifically mentioned (chronic diseases) (8.1\%)

$2.4 \%(\mathrm{CAM})$

$0.5 \%$ (endstage renal disease)

Documentation not specifically mentioned

-

-

$-$

-

-

-

-

$-$

$9.5 \%$

$10.0 \%$

$-$

$-$

$-$

aIn FGDs, we asked for only critical topics. About $67 \%$ of same or similar topics were ranked as critical nursing and midwifery clinical research topics in both the Delphi survey and FGDs. ${ }^{\text {IIn }}$ both the Delphi and scoping review, the focus was on clinical nursing and midwifery research topics (i.e., those with patient-related outcomes), thus some topics may not be included because they were not the focus of the study. ${ }^{c}$ Ranked as moderately important in Delphi survey (all unmarked topics were ranked as critically important research priorities). ${ }^{\mathrm{d}}$ Ranked as important in Delphi survey (all unmarked topics were ranked as critically important research priorities). CAM, complementary and alternative medicine; FGD, focus group discussion. 
to differ somewhat from those of nursing leadership and academic researchers, we found many similarities, which reinforces the need for research in those areas of overlap. Also, some specific clinical topics (e.g., nil per os status, alarm fatigue, and documentation) illuminate areas of interest for future research.

Most topics had some element of HSR and/or nursing shortages, which had a pervasive impact on nurses and midwives in all three countries. Participants in all groups discussed whether improving nurses' conditions and staffing levels could affect patient care. The overwhelming shortage of resources, not only in terms of supplies or gaps in healthcare infrastructure that created difficulty for nursing practice, but also to the shortage of time for nurses to provide safe and quality care created by the lack of human resources was frequently discussed. Nursing workforce was the most frequently coded theme and important codes included nursing roles and staffing levels of nurses. Participants showed interest in knowing the best patient ratios to reduce documentation errors and the effect of the nursing shortage on nurses' personal wellbeing.

The knowledge gaps theme illustrated another facet of the need for systemic changes to nursing education. Many nurses had difficulty articulating what evidence-based practice meant, showing a gap in training and continuing education. Additionally, some topics arose, like evidence of the efficacy of the human papillomavirus vaccine (mentioned in the Saudi Arabia FGD), that are already well researched, suggesting a lack of availability or dissemination of current research.

\subsection{Quantitative Comparison to Previous Research}

Sixty-seven percent of research topics matched between the FGDs and the recent Delphi survey [14], suggesting that overall, nursing leadership and clinical staff have similar ideas about critical topics for building a solid evidence base. While nearly $66 \%$ of topics discussed in FGDs were not revealed in the scoping review, about $24 \%$ may not have been identified because they did not have specific patient-related outcomes. However, of those topics with patientrelated outcomes, 19 (50\%) were not identified in the scoping review, suggesting that clinical nurses truly identified gaps in existing literature that warrant further research.

\subsection{Limitations}

This study had several limitations. The sample only represents three of the 22 EMR countries (Figure 1), and only one or two FGDs were conducted in each country. Although eight countries originally agreed to participate, only three were able due to regional conflicts, displacement of researchers, and political turmoil, which reflects the profound complexities facing healthcare and nursing practice in this geographical area. However, they represent a diverse sample; Saudi Arabia and Oman are low-child, -adult mortality, while Egypt is a high-child, -mortality country [21]; yet many topics were replicated in all three, suggesting some regional generalizability and saturation of data despite heterogeneity between countries. Further exploration of between-country differences and a larger sample would enhance understanding of regional clinical research priorities. Differences in coding topics between the FGDs, the Delphi survey, and scoping review $[12,14]$ may have decreased the ability to accurately compare data, and these results should be interpreted with caution. Nevertheless, triangulation of the studies lends a broader understanding of the current state of regional nursing science.

\section{CONCLUSION}

Focus group discussions were analyzed to understand clinical research priorities as seen by nurses and midwives in the EMR. Major issues across countries were related to the nursing workforce, stemming from educational gaps and nursing shortage; skill mix explorations into these priorities could reveal ways to alleviate the healthcare burden and improve nursing practice and patient outcomes. Future quantitative studies can help to address the needs discovered in this qualitative, exploratory study (such as improving the implementation of research into practice).

Priorities and themes identified from this study and the Delphi survey together suggest a regional agenda should focus on HSR (specifically, reducing the nursing shortage and standardizing training and education) and culturally relevant patient-related clinical topics. Moreover, research on nursing shortages can have global applications, as issues of undefined or unclear scope of practice, lack of autonomy, variable educational requirements, workload, and remuneration are commensurate with problems identified throughout all WHO regions [22]. Finally, this study suggests that while there may be concern about whether administrators setting research agendas would align with those of frontline clinicians, they may be assuaged by the fact that clinical nurses identified similar priorities to those of nurse administrators; both groups identified topics that represented gaps in the literature.

\section{ACKNOWLEDGMENTS}

This study was funded by Columbia University School of Nursing.

\section{CONFLICTS OF INTEREST}

The authors have no conflicts of interest to declare.

\section{REFERENCES}

[1] United Nations. Goal 3; Good health and well-being. United Nations Dev Program. 2017. Available at: http://www.undp.org/ content/undp/en/home/sustainable-development-goals/goal3-good-health-and-well-being.html. (Accessed September 10, 2017).

[2] Chalmers I, Bracken MB, Djulbegovic B, Garattini S, Grant J, Gülmezoglu AM, et al. How to increase value and reduce waste when research priorities are set. Lancet 2014;383;156-65.

[3] World Health Organization. Global strategic directions for strengthening nursing and midwifery 2016-2020. Geneva: WHO; 2016.

[4] Alinier G, Platt A. International overview of high-level simulation education initiatives in relation to critical care. Nurs Crit Care 2014;19;42-9. 
[5] United Nations High Commissioner for Refugees. Geneva: UNHCR; 2016.

[6] Melnyk BM, Fineout-Overholt E. Evidence-based practice in nursing \& healthcare: a guide to best practice. Philadelphia: Wolters Kluwer Health/Lippincott Williams \& Wilkins; 2011.

[7] World Health Organization. WHO Nursing and Midwifery Progress Report 2008-2012. Geneva: WHO; 2013.

[8] Sun C. Clinical Nursing and Midwifery Research in Southern and Eastern African Countries. (PhD in Nursing), Columbia University, New York, NY. Retrieved from https://academiccommons.columbia.edu/doi/10.7916/D8251HKK; 2015.

[9] World Health Organization. Quantifying environmental health impacts: Country groupings. Available at: http://www.who. int/quantifying_ehimpacts/global/ebdcountgroup/en/.(Accessed May 22, 2016).

[10] Tingen MS, Burnett AH, Murchison RB, Zhu H. The importance of nursing research. J Nurs Educ 2009;48;167-70.

[11] Powell K. Nursing research: Nurses know best. Nature 2015; 522;507-9.

[12] Alhusaini M, Sun C, Larson E. Clinical nursing and midwifery research in Middle Eastern and North African Countries: a scoping review. J Health Specialties 2016;4;238-45.

[13] Cimiotti J, Quinlan P, Larson E, Pastor D, Lin S, Stone P. The Magnet Process and the perceived work environment of nurses. Nurs Res 2005;54;384-90.
[14] Sun C, Dohrn J, Oweis A, Huda AH, Abu-Moghli F, Dawani H, et al. Delphi survey of clinical nursing and midwifery research priorities in the eastern mediterranean region. J Nurs Scholarsh 2017;49;223-35.

[15] Morgan DL, Krueger RA, King JA. The focus group kit. Volumes 1-6. Thousand Oaks, CA: Sage; 1998.

[16] Rabiee F. Focus-group interview and data analysis. Proc Nutr Soc $2004 ; 63 ; 655-60$

[17] Tong A, Sainsbury P, Craig J. Consolidated criteria for reporting qualitative research (COREQ): a 32-item checklist for interviews and focus groups. Int J Qual Health Care 2007;19;349-57.

[18] Shenton AK. Strategies for ensuring trustworthiness in qualitative research projects. Educ Inf 2004;22;63-75.

[19] McHugh ML. Interrater reliability: the kappa statistic. Biochem Medica 2012;22;276-82.

[20] World Health Organization Alliance for Health Policy and Systems Research. What is health policy and systems research (HPSR)? Overview 2017. Available at: http://www.who.int/alliance-hpsr/about/hpsr/en/. (Accessed October 23, 2017).

[21] World Health Organization. Quantifying environmental health impacts: country groupings 2017. Available at: http://www. who.int/quantifying_ehimpacts/global/ebdcountgroup/en/. (Accessed September 12, 2017).

[22] Wong FKY, Liu H, Wang H, Anderson D, Seib C, Molasiotis A. Global nursing issues and development: analysis of World Health Organization documents. J Nurs Scholarsh 2015;47;574-83.

\section{APPENDIX A. QUESTIONING ROUTE AND SAMPLE CODES FROM CODEBOOK}

\section{Questioning route}

1. Tell us who you are, which unit you work in and one thing you like to do when you are not working (5 min).

2. The interventions we practice as nurses and midwives are based on research. We call this evidence-based practice. What kinds of problems or situations that affect your patients have you encountered in your clinical work as a nurse that you could be solved through scientific evidence or research? (15 min).

3. Based on your experiences, which research topics do you think are most critical for nurses and midwives to research now in order to help patients? (10 min).

4. Based on the problems you have been describing, what specific research questions do you see as critical in order to help patients or improve care? (10 min).

5. (Moderator summarizes what was said in 2 minutes or 3 minutes and then asks) Is there anything I missed about what is really most important for research that will help nurses and midwives to improve patient outcomes? $(5 \mathrm{~min})$.

\begin{tabular}{|c|c|c|}
\hline Code & Definition & Examples \\
\hline Communication & $\begin{array}{l}\text { Description of } \\
\text { communication between } \\
\text { healthcare worker and } \\
\text { patient, and between } \\
\text { healthcare workers }\end{array}$ & $\begin{array}{l}\text { There is also another problem you need to consider, which is communication between ourselves; it is very bad. } \\
\text { Also communication between nursing and patients. (Egypt Focus Group 2a, pp. 16-17) } \\
\text { "I feel there is lack of communication sometimes with doctor and patient. Because sometimes patient will come } \\
\text { out and still he's asking us like 'why this doctor collecting this blood for us?' So many patients, ask me. I cannot } \\
\text { interfere because this is his doctor's treatment. So I think there is a big gap in communication between doctor and } \\
\text { patient. And I hope to improve this [by providing] a clear picture for the patient." (Oman Focus Group, pp. 458-66) }\end{array}$ \\
\hline Culture & $\begin{array}{l}\text { Relevance or cultural } \\
\text { sensitivity of care }\end{array}$ & $\begin{array}{l}\text { For our culture, Islamic culture, this is something we have to modify and research and then we have to reapply it ... } \\
\text { (Oman Focus Group, pp. 164-8) }\end{array}$ \\
\hline Policy & $\begin{array}{l}\text { Policy and protocols } \\
\text { for care }\end{array}$ & $\begin{array}{l}\text { Working in a neurosurgery ward, we have to sometimes restrain the patients. But, we do not have the restraint } \\
\text { policies in the hospital. (Oman Focus Group, pp. 253-7) } \\
\text { "... when a case is admitted, there should be a protocol to screen for infection and to remove invasive devices. } \\
\text { And there is no clear protocol. Some people do and some don't. That means I can accept or reject any case } \\
\text { upon admission, as there are some cases that I can't handle properly for not having suitable or enough sup- } \\
\text { plies or isolation." (Egypt Focus group 2a, pp. 10-1) }\end{array}$ \\
\hline \multirow[t]{2}{*}{$\begin{array}{l}\text { Nursing } \\
\text { workforce }\end{array}$} & $\begin{array}{l}\text { Issues related to nursing } \\
\text { shortage scope of } \\
\text { practice, workload, } \\
\text { remuneration, } \\
\text { motivation }\end{array}$ & $\begin{array}{l}\text { "The nursing team is severely suffering, receives low salaries, no recognition, and is stressed and stretched out in } \\
\text { work. Now, how do we do research, and what strategies do we establish? We need to perform testing on strategies } \\
\text { to relieve workload on nurses, focus on how they can receive better education on how to work in certain work } \\
\text { environments, how to increase interest in work and receive salaries [that] ensure good living standard." (Egypt } \\
\text { Focus Group 2b, p. 14) }\end{array}$ \\
\hline & & $\begin{array}{l}\text { "We can summarize this as burnout. [Nurses] are all burned out in work; [so they are] ignoring their duties and } \\
\text { work." (Egypt Focus Group 2a, p. 16) }\end{array}$ \\
\hline
\end{tabular}


APPENDIX B. QUANTITATIVE ASSESSMENT OF FREQUENCY OF CODES BY COUNTRY

\begin{tabular}{|c|c|c|c|c|}
\hline \multirow{2}{*}{ Code } & \multicolumn{3}{|c|}{ Number of times used } & \multirow{2}{*}{$\begin{array}{l}\text { Total number of } \\
\text { times used }\end{array}$} \\
\hline & Egypt & Oman & Saudi Arabia & \\
\hline Nursing Workforce & 100 & 22 & 9 & 131 \\
\hline Health Systems Research & 60 & 9 & 8 & 77 \\
\hline Quality/Safety & 42 & 9 & 11 & 62 \\
\hline Policy & 29 & 13 & 2 & 44 \\
\hline Healthcare Infrastructure & 29 & 10 & 1 & 40 \\
\hline Infection Control & 17 & 12 & 4 & 33 \\
\hline Lack of Resources & 22 & 8 & 2 & 32 \\
\hline Communication & 13 & 6 & 9 & 28 \\
\hline Women's Health & 0 & 1 & 26 & 27 \\
\hline Using Evidence & 12 & 7 & 7 & 26 \\
\hline Medications & 9 & 6 & 7 & 22 \\
\hline Pediatrics & 3 & 15 & 2 & 20 \\
\hline Mental Health of Nurses & 11 & 4 & 2 & 17 \\
\hline Patient Education & 2 & 4 & 11 & 17 \\
\hline Pain Management & 2 & 10 & 2 & 14 \\
\hline Public Awareness and Safety & 2 & 4 & 7 & 13 \\
\hline Mental Health & 3 & 6 & 3 & 12 \\
\hline Length of Stay & 4 & 7 & 0 & 11 \\
\hline Complementary/Alternative Medicine & 0 & 2 & 8 & 10 \\
\hline Documentation & 10 & 0 & 0 & 10 \\
\hline Nutrition & 8 & 0 & 1 & 9 \\
\hline Patient Participation in Care & 4 & 3 & 1 & 8 \\
\hline Obesity & 7 & 0 & 0 & 7 \\
\hline Palliative Care & 3 & 4 & 0 & 7 \\
\hline Public Perception of Nursing & 4 & 0 & 3 & 7 \\
\hline Culture & 1 & 5 & 0 & 6 \\
\hline Road Traffic Accidents & 0 & 6 & 0 & 6 \\
\hline Burns & 0 & 5 & 0 & 5 \\
\hline Healthcare Costs & 3 & 2 & 0 & 5 \\
\hline Wound Care & 0 & 1 & 4 & 5 \\
\hline Nil Per Os (NPO) & 0 & 4 & 0 & 4 \\
\hline Sexually Transmitted Infections & 0 & 0 & 4 & 4 \\
\hline Access & 1 & 1 & 0 & 2 \\
\hline Alarm Fatigue & 2 & 0 & 0 & 2 \\
\hline Explosions (“Bombings") & 0 & 2 & 0 & 2 \\
\hline Primary Care & 0 & 2 & 0 & 2 \\
\hline Emergency Preparedness & 1 & 0 & 0 & 1 \\
\hline Geriatrics & 1 & 0 & 0 & 1 \\
\hline Per-FGD Subtotals & 405 & 190 & 134 & 729 \\
\hline
\end{tabular}

